\title{
Test of the Precision and Accuracy of the ShapeAccelArray Sensor
}

\author{
Martin Štroner ${ }^{\mathrm{a}}$, Rudolf Urban ${ }^{\mathrm{a}}$, Jan Balek ${ }^{\mathrm{a}, \mathrm{b}}$ \\ ${ }^{a}$ Department of Special Geodesy, The Czech Technical University in Prague, Thákurova 6, \\ Prague 6, Czech Republic, 16629 \\ bInstitute of Rock Structure and Mechanics of the ASCR, v. v. i., V Holešovičkách 94, \\ Prague 8, 18200 \\ martin.stroner@fsv.cvut.cz, rudolf.urban@fsv.cvut.cz, balek@irsm.cas.cz
}

\begin{abstract}
Measurand MEMS ShapeAccelArray sensors are in many ways unique and new equipment, which allows to determine the relative displacements and deformations in other ways than before. The instruments are described, tested and compared with existing instrumentation in a small number of articles, but with exception of one it was not tested from engineering surveying point of view, where the exact precision and accuracy knowledge is a basis for the further use. The result of the experiments is the accuracy of measuring changes by the sensor itself, the pros and cons of its use and some features of its design, which is good to know before using it.

There were designed and performed practical tests to determine the relative and absolute measurement accuracy of the sensor deformations measurement in all three possible types of use, namely the vertical installation, the horizontal installation and the measurement of convergence.

The results show high accuracy of the sensor tested, which is under certain conditions even considerably higher than that given by Measurand.
\end{abstract}

Keywords: ShapeAccelArray sensor; precision test; accuracy test.

\section{Introduction}

Sensors and other measuring instruments forms currently very large group, which offers wide possibilities of usage and generally is used in more interdisciplinary way. Not only a traditional surveying instruments (as total stations, GNSS receivers or 3D technology of (laser) scanning and photogrammetry) are used in engineering surveying, but also various geotechnical sensors such as inclinometers, pendulums, stringed potentiometers etc. An advantage of geodetic measurement is the possibility to determine the absolute displacements in a suitably selected coordinate system at such a long distance, that the points defining this reference system are located outside the area of deformation (e.g. for monitoring of the buildings' behavior or for the determining the effect of extensive building operations). Geotechnical sensors provide often faster and simpler measurement than geodetic, but displacements are only relative (tilt, extension etc.).

One of the last new types of sensors is SAA (ShapeAccelArray) produced by Measurand Inc. This sensor is actually an array of inclinometers and enables variable usage due to the possible long length (up to $100 \mathrm{~m}$ ) and also different variants of installation. The sensor was 
tested from the geodetic point of view for the purpose of measuring of the relative changes in this article. Results of the first part of tests is presented here, where the testing was carried out without the cover consisting of plastic pipes, in which the sensor should be placed during the installation according to the manufacturer's instructions. The intention of the tests was to determine the measurement accuracy of the sensor itself, without the further limiting influence of the housing. There is also not only hypothetical possibility of the use a sensor for determining e.g. deformation of a structure without the presence of a plastic pipe and with the physically hard mounting to a structure.

The use and the testing of the sensor is described in commonly available publications. Many of them are presented directly at the manufacturer's website ([7], [12], [11], [9], [5]), others were published in the independent scientific press.

Dasenbrock [7] describes the use of the SAA sensor while monitoring a landslide that cause displacements and damage of the road. The important information is that used SAA measuring systems after 900 days of use and frequency of reading every 6 hours were still functional. The development of displacements according to the time and depth was described using the SAA sensors. The aim of this research was to create a model of terrain, including the composition of the soil and install the SAA near expected slip surface for early detection of landslides, thus creating landslide early warning system (to close the road traffic in time). Analysis of accuracy was not done.

Thurlow [12] describes the use of the SAA for sheeting walls monitoring. For this measurement only inclinometers were previously used. Presentation show graphs as a result of the comparison of those two approaches. The graph suggests that the values obtained by the two methods are comparable. From the grid of a graph differences about $2 \mathrm{~mm}$ can be inferred. Presentation not contains a comparative table or other comparison of the difference of measurement results.

Article by Measurand [9] describes the use of SAA on the railway line on the coast on unstable subsoil and article by Measurand [5] deals with the use of SAA for dam's deformation measurement. The SAA is there compared with the pendulum methods. The article indicates that the measured data by both methods show compliance. The accuracy of pendulums is given by a value of $0.3 \mathrm{~mm}$. The article does not indicate anything about the accuracy of the SAA and does not include an evaluation of the differences from the measured data.

Abdoun [1] compared total displacements determinated by the SAA and by the inclinometer in real-time monitoring. The results of the comparison were shown in the graph only. The graph shows a comparable trend in both kinds of measurement. The author indicates the value for SAA "repeatability", which during 8 months (hundreds of measurements) reaches value better than $1.5 \mathrm{~mm}(0.05 \mathrm{~mm} / \mathrm{m})$. Probably the same set of observations is also used in article [3] dealing with the development of the wireless SAA.

Abdoun also [2] presented a comparison of the SAA and traditional geotechnical methods (potentiometer and a tilt sensor and accelerometer) when using of a Shape-Acceleration Array (SAA) to instrument full-scale laminar container tests at the University of Buffalo. The results are graphs comparing measurements of acceleration and lateral displacement using traditional methods and SAA. The results of comparison based on the published graphs and author's description are comparable. Authors does not provide any analysis of measurement accuracy or analysis of comparable result in numeric values. 
Danisch [6] presented a results of the comparison of the SAA technology and robotic total station results. The publication describes advantages of geodetic and geotechnical measurements using a combination and interconnection of various kinds of surveying methods and sensors (such as SAA, robotic total station (RTS), global navigation satellite system (GNSS)). The experiment was based on the realization of simulated displacement and monitoring of it by different sensors. By SAA was monitored lateral shift only, part of the results was included in a table comparing the expected and measured shift. By the SAA technology the $19 \mathrm{~mm}$ shift was measured (20 mm expected), by the RTS the $20 \mathrm{~mm}$ and by the GPS GNSS the $19 \mathrm{~mm}$ shift was determined. In conclusion the author without further justification indicates that the SAA is able to provide a millimetre results.

Using the SAA sensor within the system swissMon was described by Meyer and Schütz [10]. This system analyses and displays the data measured by the geodetic and geotechnical sensors in a railway tunnel in Zurich. The SAA is placed in the inner covering of the tunnel for monitoring the change of its shape. The authors again do not indicate anything about the accuracy of the SAA, even in comparison with other methods used for determining of the change of the shape of a tunnel.

Hendry et al. [8] presented the use of the SAA during the displacements' monitoring of railway embankment based on the unstable peat soil. Again, this is an example of usage, but the measurement accuracy of the SAA is described only by values given by the manufacturer.

An interesting comparison of geotechnical and geodetic relative displacements measuring methods is published by Beran et al. [4]. A comparison between the SAA in the near vertical installation with a geodetic measuring system formed by the robotic total station with automated data collection and processing is presented there. Part of the test was the verification of the consistency of the SAA in the form of double measurements. RTS measurement was carried out during three consecutive days, control points were signaled by the miniprisms. SAA measurement was carried out for 2 months before the start of data collection by total station. During the measurement using the SAA was determined a shift 2 $\mathrm{mm}$ on the top point. Simultaneous measurement of both systems was carried out during the day and at night under different temperature conditions. The measurement was corrected to suppress the effect of temperature changes (temperature measurement accuracy was $0.06^{\circ} \mathrm{C}$ ). Total station measurements were conducted in 3 sets and data collection of one epoch was always 30 minutes. Data collection with using the SAA was quicker (about 10 minutes), but the measurement was programmed to begin at the same time using both methods. Graphs of the top point's movement are published in the article, comparing the results of the SAA and RTS measurements. The graph shows that the movement trend of two methods is the same. Furthermore, there are published graphs comparing the measured displacements in dependence on time. The standard deviation of the differences between displacements SAA and the RTS was in all cases smaller than $1 \mathrm{~mm}$.

The influence of location and torsion of the individual segments of SAA on the results of measurement is solved by Danisch et al. [6]. Authors describe the development of the SAA as a flexible instrument with a smaller diameter of cover than the traditional inclinometers, which are placed in a PVC tube with a minimum diameter of $27 \mathrm{~mm}$. There is a test of sensor installed in the shape of a helix.

The SAA sensor was practically used in many different works according to available pub- 

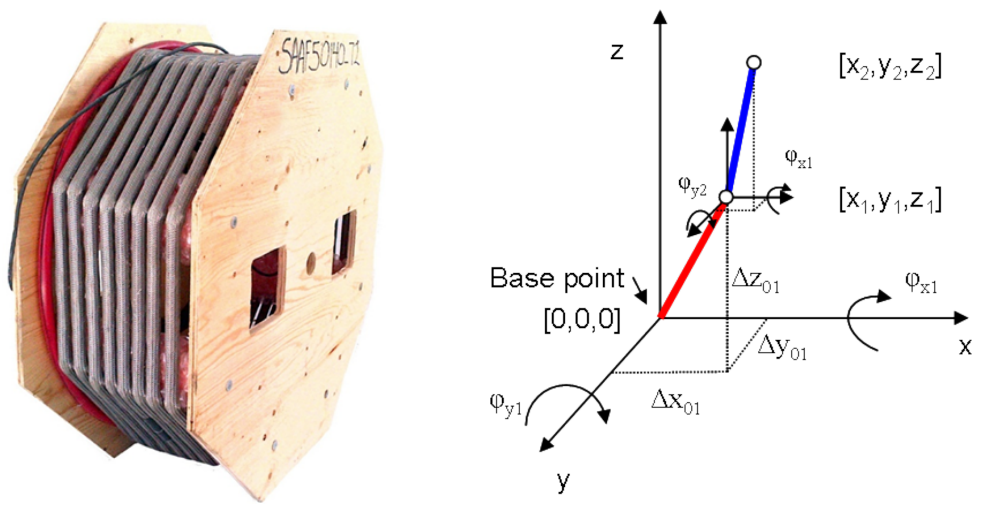

Figure 1: (a) SAA sensor on a reel. (b) Tilts and positions of SAA elements

lications, but the displacements' real accuracy determination testing has not been done so far. The analysis carried out by Beran [4] is the only exception, but there is the determined accuracy slightly devalued by comparing method, which is not significantly more accurate, as is appropriate. There is also a problem with method of installation of the sensor where the actual plastic packaging can considerably influence the determined changes.

\section{Principles of operation and use of the sensor}

The SAA is (according to a manufacturer's website www.measurandgeotechnical.com) a sensor for monitoring deformation in the field. Whole monitoring system consists of the sensor and the reading device, standard PC computer with Microsoft Windows operating system or a datalogger can be used. A Measurand interface is required between an SAA and the datalogger or a computer, also a specific Measurand software for reading and registering of the measured data to the PC is needed.

The SAA sensor itself consists of rigid segments separated by flexible joints. Each segment is equiped with triad of accelerometers and some of the segments also include temperature sensor. Triaxial MEMS (Micro-Electro-Mechanical Systems) gravity sensors measure tilt in each individual segment. Coordinates of each joint are computed relative to the one of the endpoints from measured tilt and known length of each segment. The length of each segment is estimated using calibration lengths and registered temperatures. SAAs produce data equivalent to inclinometer data (Fig. 1b).

Each SAA is a fully-calibrated measuring instrument with a length of up to 100 meters. All microprocessors in the array share the same digital communication line. Segments can be 305 $\mathrm{mm}$ or $500 \mathrm{~mm}$ long, maximum number of the segments is 200. It is limiting a maximum length up to $100 \mathrm{~m}$ for $500 \mathrm{~mm}$ segments or $60.96 \mathrm{~m}$ for $305 \mathrm{~mm}$ segments.

The sensor joint diameter in extension is $25 \mathrm{~mm}$, in compression $27 \mathrm{~mm}$, maximum joint bend angle is $45^{\circ}$, installation temperature is between $-5^{\circ} \mathrm{C}$ and $60^{\circ} \mathrm{C}$, operating temperature is between $-35^{\circ} \mathrm{C}$ and $60^{\circ} \mathrm{C}$, sensor is waterproof up to $980 \mathrm{kPa}$ water pressure. The long-term accuracy relative to the starting shape is specified to be $\pm 1.5 \mathrm{~mm}$ for $32 \mathrm{~m}$ SAA by the manufacturer, a short-term accuracy is given by the value $\pm 0.5 \mathrm{~mm}$ for $32 \mathrm{~m}$ SAA. The specifications in full detail can be found in www.measurandgeotechnical.com. The sensor itself on transportation reel is on Fig. 1a. 


\section{Experiments and results}

To determine the accuracy of measurement of changes by the sensor itself without influence of installation housing, the tests corresponding to the fundamental methods of installation were designed, namely horizontal, vertical and circular circular one (e.g. to measure changes in shape of the tunnels). For determining of the shape changes by the sensor it is not easy to find a suitable reference method, because the expected accuracy is at a shorter distance far below $1 \mathrm{~mm}$. Moreover, on the sensor are no usable reference points that could be geodetically determined. Absolute tests to determine the actual shape of the sensor are practically impossible due to the protective covering. Tests were therefore designed as relative, so that the change was in a specific and well defined known direction and simultaneously determinable by comparative method with the higher accuracy. For the tests a sensor of the length of $15.250 \mathrm{~m}$ consisting from the 50 segments of length $305 \mathrm{~mm}$ was used. Because the measurements were made at different locations (at different distances from the beginning) of the sensor, for easier comparison of the determined precision the standard deviations were recalculated to the standard deviation per one segment $s_{0}(1)$. It should be noted that this is a standard deviation and the standard deviation of the measurement at the end of the n-th segment is determined as $s_{0} \cdot n$.

$$
s_{0}=\frac{s}{\sqrt{n}}
$$

As the first, the circular assembly test was carried out testing the longitudinal and the transversal accuracy and precision in altogether, then the separated horizontal and vertical installation tests were carried out.

\section{Experiment determining the accuracy of measurement of displacements in a circular assembly}

During the experiment, the sensor was installed on approximately vertical structure in the shape of the convergence profile and placed on the wooden frame. Sensor was fixed to a wall in the cellar of the Faculty of Civil Engineering in Prague (Fig. 2). In this position, it was difficult to cause the controlled displacements with known size without changing the orientation of the segment. Therefore, the four segments were fixed using sleeves (painted in red). Fig. 3 installation of the fixed and nonfixed segments is shown at Fig. 5 and Fig. 6).

Within the test the position of the intermediate (freestanding) segments was slightly changed (in millimeters to centimeters). In the case of error-free measurements should the coordinate of endpoints of fixed segment should be determined identically, the differences between the individual stages are the products of measurement errors. It was measured 15 stages, in each stage always five times in immediate succession - without changing the position of the sensor (2). Each of these measurements ( $x, z)$ always consisted of the average of 1000 repeating (100 measurement averaging in sensor, 10 readings). The results are two types - standard deviation of repetitions in each stage - respectively its course depending on the order of the segment $(3,4)$ and standard deviations of stable points of the fixed segments $(5)$. 


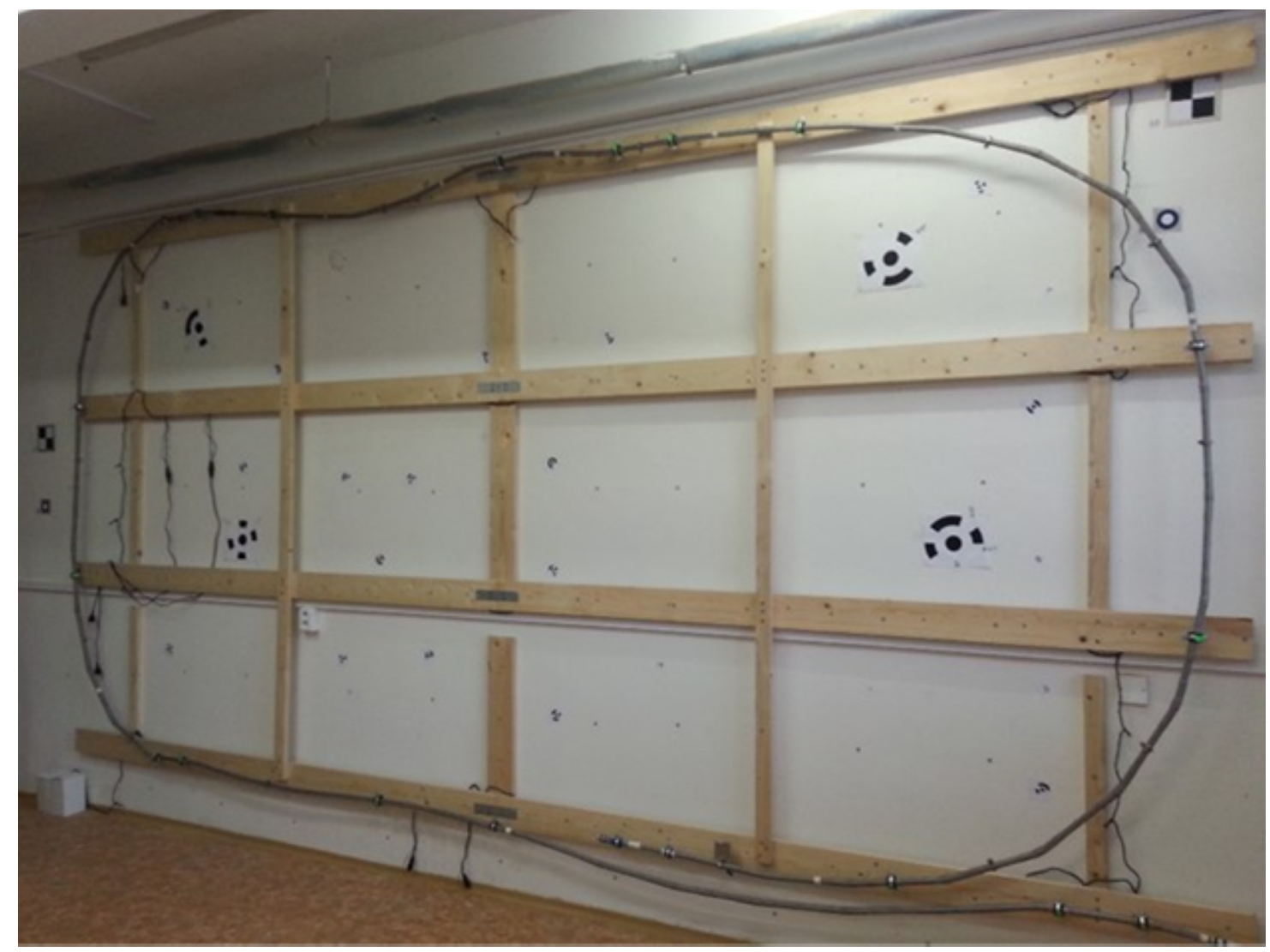

Figure 2: SAA in a circular assembly

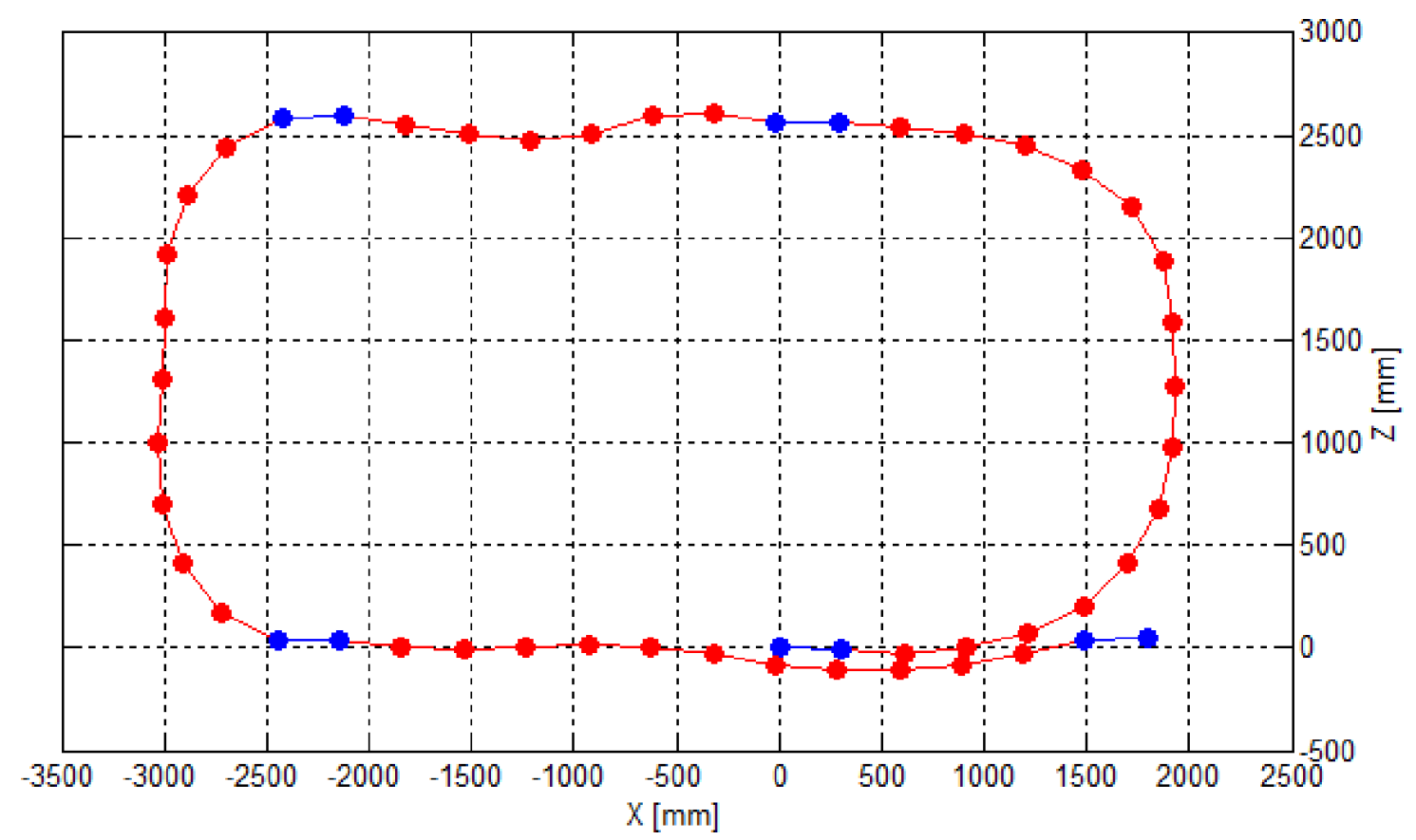

Figure 3: Circular assembly - dimensions (in $\mathrm{mm}$ ) and stabilized segments (painted orange) 


$$
\begin{aligned}
\bar{x} & =\frac{\sum_{i=1}^{5} x_{i}}{5} ; \quad \bar{z}=\frac{\sum_{i=1}^{5} z_{i}}{5} \\
{ }^{j} S_{x} & =\sqrt{\frac{\sum_{j=1}^{5}\left(x_{i}-\bar{x}\right)^{2}}{5-1} ; \quad{ }^{j} S_{z}=\sqrt{\frac{\sum_{j=1}^{5}\left(z_{i}-\bar{z}\right)^{2}}{5-1}} ;} \\
{ }^{r} S_{x} & =\sqrt{\frac{\sum_{j=1}^{15}{ }^{j} S_{x}^{2}}{15} ; \quad{ }^{r} S_{z}=\sqrt{\frac{\sum_{j=1}^{15}{ }^{j} S_{z}^{2}}{15}} ; \quad{ }^{r} S=\sqrt{{ }^{r} S_{x}^{2}+{ }^{r} S_{z}^{2}}} \\
\overline{\bar{x}} & =\frac{\sum_{i=1}^{15} \bar{x}_{i}}{15} ; \quad \overline{\bar{z}}=\frac{\sum_{i=1}^{15} \bar{z}_{i}}{15} ; \\
{ }^{f} S_{x} & =\sqrt{\frac{\sum_{i=1}^{15}\left(\bar{x}_{i}-\overline{\bar{x}}\right)^{2}}{15-1}} ; \quad{ }^{f} S_{z}=\sqrt{\frac{\sum_{i=1}^{15}\left(\bar{z}_{i}-\overline{\bar{z}}\right)^{2}}{15-1}} ; \quad{ }^{f} S=\sqrt{{ }^{f} S_{x}^{2}+{ }^{f} S_{z}^{2}}
\end{aligned}
$$

Standard deviations determined from repeated measurements were quadratically averaged (4) and shown in Fig 4. The graph of standard deviation of repetitions (rsx is standard deviation in coordinate $\mathrm{X},{ }^{r} s_{z}$ is standard deviation in coordinate $\mathrm{Z},{ }^{r} s$ is standard deviation in position (2) , Polyg. $\left({ }^{r} s\right)$ is ${ }^{r} s$ fitted by 2 nd degree polynomial.

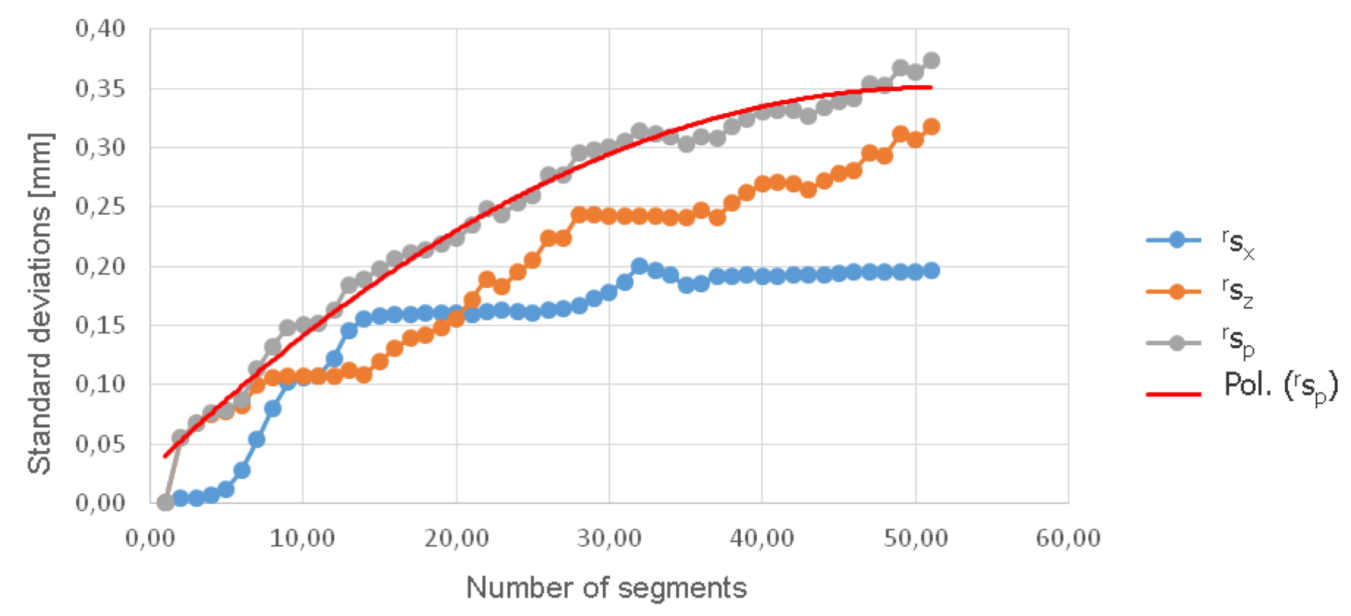

Figure 4: The graph of standard deviation of repetitions (rsx is standard deviation in coordinate $\mathrm{X},{ }^{r} s_{z}$ is standard deviation in coordinate $\mathrm{Z},{ }^{r} s$ is standard deviation in position (2), Polyg. $\left({ }^{r} s\right)$ is ${ }^{r} s$ fitted by 2 nd degree polynomial.

The average standard deviation of repetitions (for a mean of 1000 measurements per one segment) is ${ }^{r} s_{0}=0.052 \mathrm{~mm}(1,4)$. (For comparison with the following values the result corresponding to 5000 measurements mean is ${ }^{r} s_{0}=0.023 \mathrm{~mm}(1,4)$, for further evaluation each five measurements in stage were averaged). The Fig. 4 shows that the development of standard deviations in position ${ }^{r} s$ corresponds very well with the increase in square root of the number of segments. Progress of standard deviations in the individual coordinates may seem confusing - growth is stopped in some areas. This is due to fact, that the measurement of the longitudinal direction of is not done (length of the segments is considered to be fix) and therefore, . if So if some of the segments are parallel to the coordinate axis, the measurement errors growth in the perpendicular direction only. Each standard deviation in the graph is determined from 60-redundant values. 


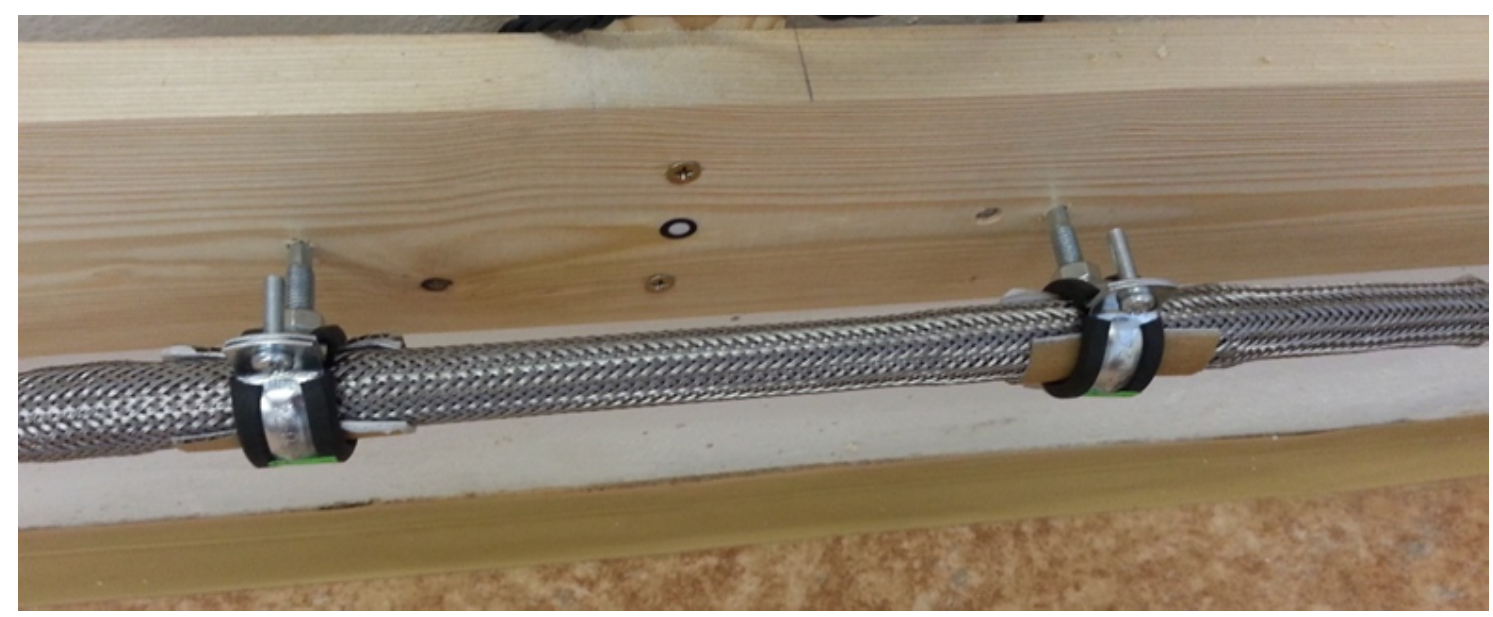

Figure 5: Fixation of segment

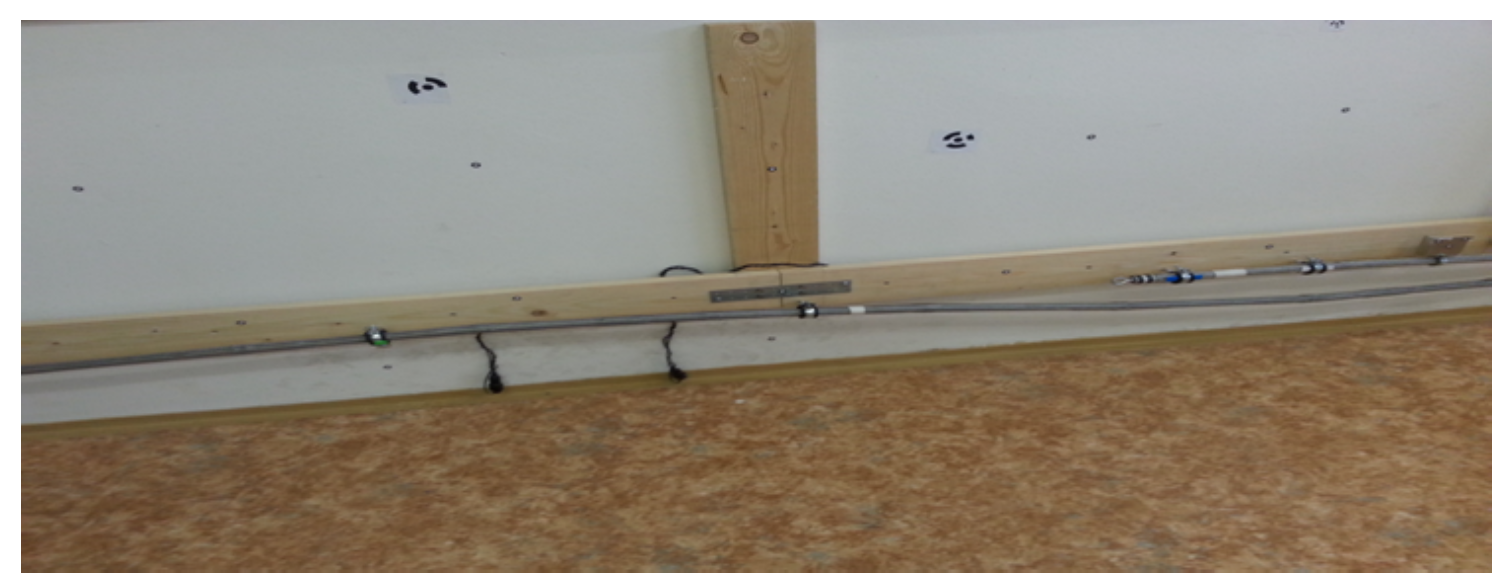

Figure 6: Ordinary (floating) installation of the sensor

When evaluating the stable segments the standard deviations were determined for all fixed points - always to both ends of the segment (excluding the point 0). Stabilized points were $0,1,17,18,25,26,36,37,49$, and 50 . The five measurements in each stage was were always averaged, i.e. standard deviation applies to the average from 5000 measurements.

In Tab. 1 are the standard deviations of determination of coordinates of fixed points. The results of determination of displacement accuracy are in Tab. 2, where it can be practically seen the identical estimation of precision in position in the range of $0.04 \mathrm{~mm}-0.05 \mathrm{~mm}$. (These values cannot be averaged, they are dependent).

Standard deviation determined at the last point includes the errors of all segments and therefore it is the most trustworthy. Others standard deviations are given for to show the development of precision with increasing distance from the origin. 
Table 1: The standard deviations of determination the coordinates of individual stable points of the sensor $\left({ }^{f} S_{x}\right.$ - overall for coordinate X, ${ }^{f} S_{z}$ - overall for coordinate Z, ${ }^{f} S_{x_{0}}$ - per one segment for coordinate $\mathrm{X},{ }^{f} S_{z_{0}}-$ per one segment for coordinate Z)

\begin{tabular}{|c|c|c|c|c|c|c|c|c|c|}
\hline $\begin{array}{c}\text { Standard } \\
\text { deviation } \\
{[\mathrm{mm}]}\end{array}$ & \multicolumn{9}{|c|}{ Point } \\
\cline { 2 - 11 } & 1 & 17 & 18 & 25 & 26 & 36 & 37 & 49 & 50 \\
\hline \hline${ }^{f} S_{x}$ & 0.002 & 0.120 & 0.120 & 0.119 & 0.120 & 0.208 & 0.207 & 0.224 & 0.224 \\
\hline${ }^{f} S_{x_{0}}$ & 0.002 & 0.029 & 0.028 & 0.024 & 0.023 & 0.035 & 0.034 & 0.032 & 0.032 \\
\hline${ }^{f} S_{z}$ & 0.031 & 0.166 & 0.188 & 0.173 & 0.171 & 0.222 & 0.232 & 0.237 & 0.232 \\
\hline${ }^{f} S_{z_{0}}$ & 0.031 & 0.040 & 0.044 & 0.035 & 0.033 & 0.037 & 0.038 & 0.034 & 0.033 \\
\hline
\end{tabular}

Table 2: The standard deviation of determination of the position $\left({ }^{f} S_{p}-\right.$ overall, ${ }^{f} S_{p_{0}}-$ per one segment)

\begin{tabular}{|c|c|c|c|c|c|c|c|c|c|}
\hline $\begin{array}{c}\text { Standard } \\
\text { deviation } \\
{[\mathrm{mm}]}\end{array}$ & \multicolumn{10}{|c|}{ Point } \\
\cline { 2 - 11 } & 1 & 17 & 18 & 25 & 26 & 36 & 37 & 49 & 50 \\
\hline \hline${ }^{f} S$ & 0.031 & 0.205 & 0.223 & 0.210 & 0.208 & 0.304 & 0.311 & 0.326 & 0.322 \\
\hline${ }^{f} S_{0}$ & $\mathbf{0 . 0 3 1}$ & $\mathbf{0 . 0 5 0}$ & $\mathbf{0 . 0 5 3}$ & $\mathbf{0 . 0 4 2}$ & $\mathbf{0 . 0 4 1}$ & $\mathbf{0 . 0 5 1}$ & $\mathbf{0 . 0 5 1}$ & $\mathbf{0 . 0 4 7}$ & $\mathbf{0 . 0 4 6}$ \\
\hline
\end{tabular}

\section{Experiment determining the accuracy of measurements of vertical displace- ments when installed horizontally}

The sensor was placed on approximately horizontal coherent surface from brushed concrete during the experiment. Start and end of segments were fixed for experiment by sandbags to prevent their undesired movement. The sensor was tested twice in a different orientation of device - differing by $90^{\circ}$ rotation (I. and II. face). During the experiment the temperature of the device was constant - approximately $19^{\circ} \mathrm{C}$. At each position, measurements on sections $19 / 18$ were made. Using a special lifter a specific section of sensor was raised by the exact distance (given by a pitch of the screw), without changing its tilt (Fig. 7). If there was a change sensor's tilt, values determined by sensor and lifter could not be compared. At first the measurement in the initial zero position of the lifter was made. Further, the height of the lifter was changed according to the number of rotations metric threads $(1 \mathrm{x}, 3 \mathrm{x}$ and $5 \mathrm{x})$. The pitch of the screw was $1.5 \mathrm{~mm}$ per one turn.

The measurement segment was thus always lifted up by 1.5, 4.5 and $7.5 \mathrm{~mm}$ (6). After each step the measurement was performed (an average of 1000 readings). At the end of experiment $19 \times 4$ and 18x4 measured values was available for both tested orientation of the instrument. For the purposes of the experiment the values determined by the lift were considered to be flawless 


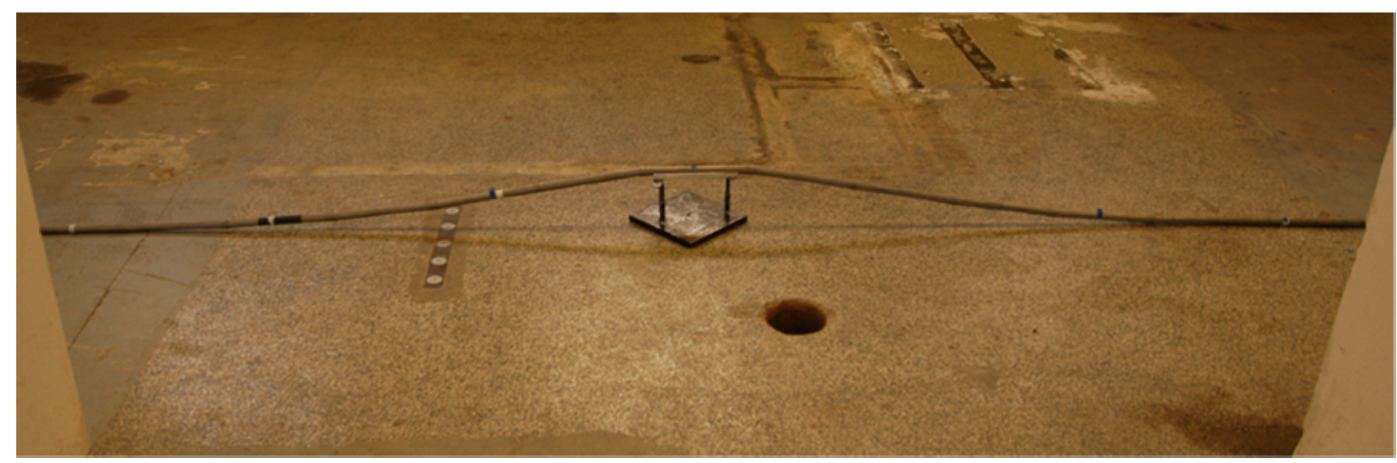

Figure 7: SAA stored in the rail, lifting trio of screws

$(\Delta z)$. Differences between lifts and measured values were considered to be true errors (7).

$$
\begin{gathered}
\Delta z^{\prime}=z_{0}^{\prime}-z^{\prime} \\
v=\Delta z-\Delta z^{\prime} \\
s_{i}=\sqrt{\frac{\sum v v}{3}} \\
s=\sqrt{\frac{\sum_{i=1}^{15} s_{i}}{15}}
\end{gathered}
$$

Testing was carried out for the $\mathrm{Z}$ coordinate only comparing with the absolute values determined by the rotation of the screw. For the calculation of the characteristics of precision the data from the lifted section was used only. Specifically hHeights of lifted section center specifically, which was calculated by an arithmetic mean of the heights of adjacent inner joints. In face I. it was obtained standard deviation of the determination of the change $0.040 \mathrm{~mm}$ per segment, in face II. $0.036 \mathrm{~mm}$ per segment. The standard deviation characterizing the accuracy of deformation depending on the magnitude of deformation were also determined, these are presented in Tab. 3. Although these values are different in hundredths of a millimeter, the differences cannot be considered statistically significant due to the small number of redundant measurements.

The result of the first experiment is the accuracy of one sensor segment $s_{0}=0.04 \mathrm{~mm}$ (for both faces) $(1,8,9)$.

Table 3: Accuracy of the deformation depending on the magnitude of deformation

\begin{tabular}{|c|c|c|c|}
\hline Deformation $[\mathrm{mm}]$ & 1.5 & 4.5 & 7.5 \\
\hline \hline$s_{0}$ in I. face & 0.038 & 0.036 & 0.044 \\
\hline$s_{0}$ in II. face & 0.032 & 0.036 & 0.040 \\
\hline
\end{tabular}

\section{Experiment determining the accuracy of measurements of horizontal dis- placements when installed vertically and fixed}

During this experiment sensor was hung up at ninth floor and run along the stairs down. The first and last segment were fixed. Between the individual stages tThe shape of the sensor was 
slightly changed (by up to $100 \mathrm{~mm}$ ) between the individual stages. There was evaluated only the change of coordinates of the last point of the sensor. 15 stages in total was measured. Each stage was composed of five separate measurements, where each consisted of an average of 1000 readings. The correct value of the position change of last point between stages is zero, the shift has been evaluated against to the first stage. There were determined the standard deviation $s_{x}=0.81 \mathrm{~mm}\left(s_{0}=0.11 \mathrm{~mm}\right)$ and $s_{y}=0.70 \mathrm{~mm}\left(s_{0}=0.10 \mathrm{~mm}\right)$ characterizing the accuracy of the average from the 5000 measurements. Standard deviations were estimated as in the test in circular assembly (5). The results of this experiment have shown following facts: If the sensor is hanging that in the vertical position when the sensor is hanging without any support and the joints are loaded by sensor's own weight, results are considerably worse than in other situations.is not suitable and there likelyThere is a probably a movement in the joints of segments (which is caused by the inappropriate installation without plastic pipe casing). Yet the measurement accuracy still achieve a value (standard deviation) in the position 1.1 mm.

\section{Experiment determining the accuracy of measurements of horizontal dis- placements when installed vertically and not fixed}

This experiment was carried out in the building B of the Faculty of Civil Engineering in Prague, where the sensor was hung up at ninth floor and run along the stairs down. The top of the sensor was mounted, the first two segments were fixed in four points. Other segments were loosely hung up. The last joint of the last segment of the sensor was attached to the engineering sledge table (Fig. 8). On the sledge table can be set the deflection from 0 to 25 $\mathrm{mm}$ in two perpendicular directions by the screw with reading with the estimated precision of $0.05 \mathrm{~mm}$.

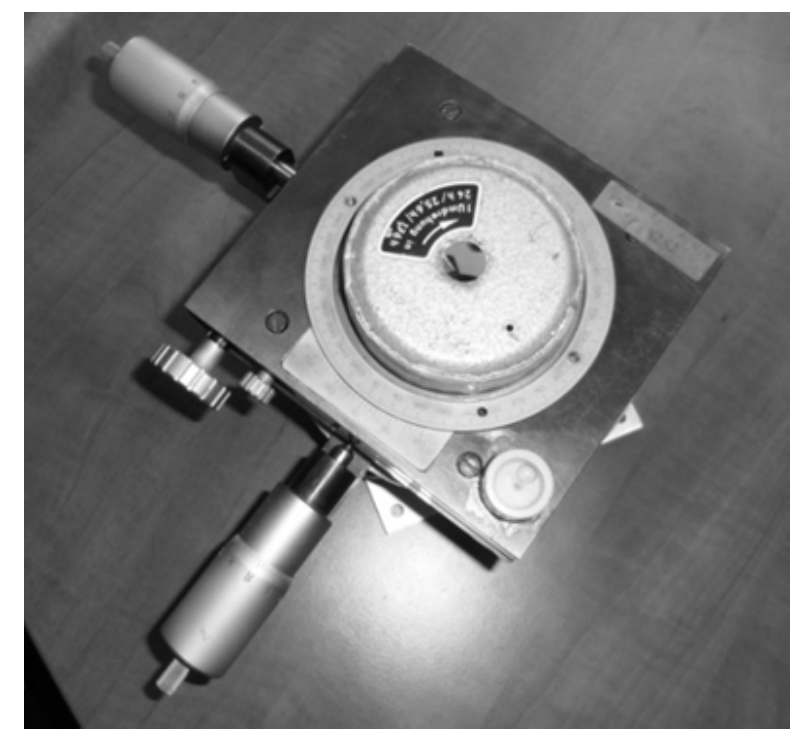

Figure 8: The engineering sledge table

The displacements were set in step $5 \mathrm{~mm}$ sequentially on both axes (Y-axis set to $0 \mathrm{~mm}$, in $\mathrm{X} 0,5,10,15,20,25 \mathrm{~mm}$ displacement in the $\mathrm{Y}$ axis to $5 \mathrm{~mm}$, repeating displacement in the $\mathrm{X}$ axis, displacement in $\mathrm{Y}$ to $10 \mathrm{~mm}$, etc.) during the testing. Hence the total 
of 36 measured values and 35 displacements versus to the start position was available for evaluation. Measurement of each stage were divided into a total of 5 parts and in each stage 1000 readings were measured. This system of measurement was realized for analysis of changes within one stage measurement, the results were the same as in the circular assembly. For the following calculations were applied always average of 5000 measurements $\left(\mathrm{X}^{\prime}\right)$. The nominal displacements and their values determined by the sensor are shown in Fig. 9 (after transformation to the coordinate system of the nominal displacements).

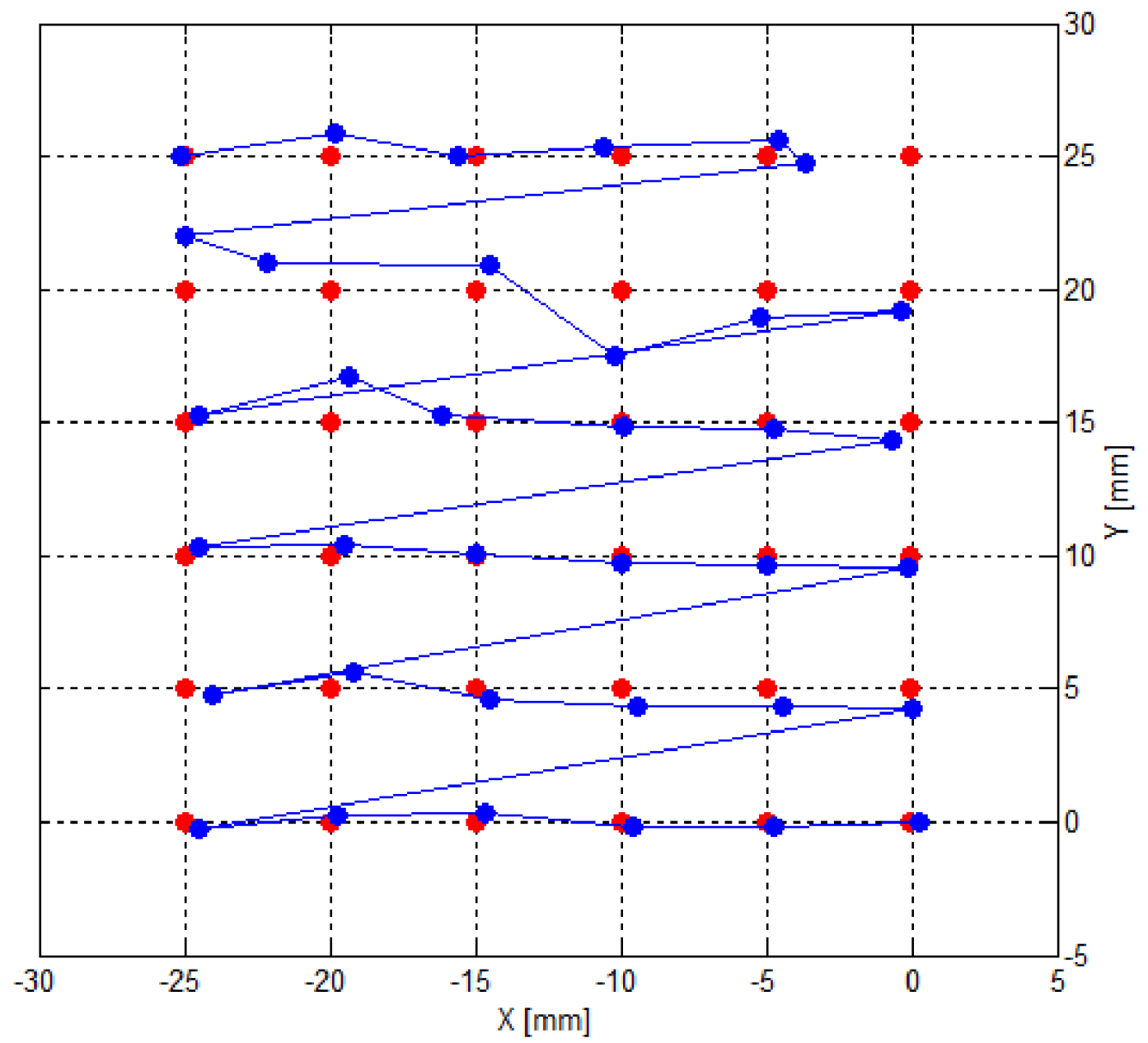

Figure 9: Nominal (red) and determined displacements (blue) in the horizontal plane

The evaluation was done in two ways. At first just the size of determined displacements were analyzed and subsequently with using $2 \mathrm{D}$ transform was discovered size and shape of displacement. As in the second part of the test (approximately after point 22) appeared obvious gross errors, further evaluation was divided into complete and partial (points 1-21). When evaluating the size of the displacement, the accuracy was determined from the difference in the size of the intended displacement between reference values. The reference value was made by the screw on the engineering sledge table. These differences are considered to be the true error. 


$$
\begin{aligned}
X & =T+R X^{\prime} \\
s & =\sqrt{\frac{\sum v v}{15}}
\end{aligned}
$$

For complete data was standard deviation of the displacement size $\mathrm{s}=0.76 \mathrm{~mm}$ (11), the standard deviation per one segment $s_{0}=0.11 \mathrm{~mm}(1)$. In contrast, 21 of the first measurement formed standard deviations $\mathrm{s}=0.38 \mathrm{~mm}$ (11) and $s_{0}^{\prime}=0.055 \mathrm{~mm}$ (1).

During the evaluation using the transformation calculation (solved by the least squares method) was used. Coordinates from engineering sledge table $\mathrm{X}$ and coordinates from the sensors $\mathrm{X}^{\prime}$ were transformed to each other (10).

For the overall evaluation the standard deviations were determined $s_{x}=0.84 \mathrm{~mm}\left(s_{x_{0}}=\right.$ $0.12 \mathrm{~mm}$ ) and $s_{y}=0.77 \mathrm{~mm}\left(s_{y_{0}}=0.11 \mathrm{~mm}\right)$. For the partial evaluation (without gross errors) $s_{x}=0.38 \mathrm{~mm}\left(s_{x_{0}}=0.054 \mathrm{~mm}\right)$ and $s_{y}=0.32 \mathrm{~mm}\left(s_{y_{0}}=0.045 \mathrm{~mm}\right)$.

In the second half of the test the disadvantage in fixation of the sensor was discovered (without encapsulation in the shroud tube). Probably when hanging due to the high weight of the sensor are the joints strained stretched. and iIt leads to a movement within the joints between the segments, causing a systematic measurement error. In the case where this phenomenon not occurs, the accuracy is very close to the previous test results.

\section{Conclusion}

The article described a measurement system formed by a special ShapeAccelArray sensor (produced by Measurand Inc. company). The measurement system is in terms of geodetic and geotechnical fields quite specific in its principle of measurement. The sensor itself consists of rigid segments separated by flexible joints. Triaxial MEMS (Micro-Electro-Mechanical Systems) gravity sensors measure tilt in each individual segment. Measuring system consists of a Measurand interface and a reading device (PC or data logger).

This measuring system was tested in all possible positions of placement (installations) without the use of plastic pipe for installation, which is recommended by the manufacturer to cover and protect the sensor.

The aim of the tests was to determine the accuracy of the sensor itself without the unknown effect of plastic pipe housing, which influences the system just by inserting the sensor into the mentioned plastic pipe.

In the case when the joints of the segments were not loaded or strained and the unwanted movements in joints were avoided, the standard deviation in determination of position was about $0.050 \mathrm{~mm}$ per segment (the average of 5000 measurements). In the case when the joints of the segments were stressed, there were (probably due to their flexible inner alignment) movements in the joints of the segments and the results were significantly worse. In both cases, the resultant standard deviation is not higher than value officially presented by Measurand $0.15 \mathrm{~mm}$ per segment.

The real accuracy of the sensor itself was not already solved in available publications with only one exception, but the comparative method used by Beran [4] was not significantly 
more accurate than the accuracy of the tested device as it should be. Moreover, the testing was carried out with the device installed in plastic pipe, which have unknown influence on the determined displacements. Therefore, comparison of the results of these two articles is inappropriate. However, Beran [4] presents standard deviation of the position about $0.5 \mathrm{~mm}$ for $4 \mathrm{~m}$ long part of the sensor, this value precisely corresponds to the value presented by Measurand (precisely calculated it could be up to $0,54 \mathrm{~mm}$ ).

Due to the construction of the sensor it is particularly necessary to consider the form of mounting. Between the segments attached to the target surface (structure) must be at least two segments free (without tension) to avoid measurement errors due to the deformation of joints. In the case of monitoring of a significantly lower required accuracy than it was achieved in presented tests, the sensor can be inserted into the plastic pipe, but this method of measurement and its accuracy was not tested.

\section{References}

[1] T. Abdoun, V. Bennett, and L. Danisch. "Innovative sensing for real-time health monitoring of geostructural systems". In: SPIE Newsroom (2007), pp. 25-27.

[2] T. Abdoun et al. "Full-Scale Laboratory Tests Using a Shape-Acceleration Array System". In: Geotechnical Earthquake Engineering and Soil Dynamics (2008), pp. 1-9. DOI: 10.1061/40975(318) 214.

[3] V. Bennett et al. "Unstable slope monitoring with a wireless Shape-Acceleration Array system". In: FMGM 200\%: Field Measurements in Geomechanics. ASCE. 2007, pp. 112.

[4] T. Beran et al. "Measurement of Deformations by MEMS Arrays, Verified at Submillimetre Level Using Robotic Total Stations". In: Geoinformatics FCE CTU 12 (2014), pp. 34-40.

[5] L. Danisch, T. Patterson, and J. Fletcher. "MEMS-Array Monitoring of a Dam". In: Proceedings of Canadian Dam Association Annual Conference. 2011, pp. 15-20.

[6] L. Danisch et al. "Fusion of geodetic and MEMS sensors for integrated monitoring and analysis of deformations". In: 13th FIG International Symposium on Deformation Measurements and Analysis, Lisbon, Portugal, May. 2008, pp. 12-15.

[7] D. D. Dasenbrock. "Automated Landslide Instrumentation Programs on US Route 2 in Crookston, MN". In: Proceedings of the Annual Conference of the Minnesota Geotechnical Society. 2010, pp. 165-185.

[8] M.T. Hendry, S. Barbour, and C.D. Martin. "An evaluation of real-time deformation monitoring using motion capture instrumentation and its application in monitoring railway foundations". In: Geotechnical Testing Journal (2011). DOI: 10.1520/GTJ103285.

[9] J. Locat et al. "An integrated mass movement monitoring system for rockslide hazard assessment at Gascons, Gaspé Peninsula, Québec: an overview". In: 63rd Canadian Geotechnical Conference and 6th Canadian Permafrost Conference, Calgary, AB. 2010, pp. 35-43.

[10] C. Meyer and S. Schütz. "swissMon-An approach to 4D Monitoring of Tunnels in Urban Environments". In: Field Measurements in GeoMechanics (2011). 
[11] K Rollins et al. "Monitoring displacement vs. depth in lateral pile load tests with shape accelerometer arrays". In: Proc. of 17th International Conference on Soil Mechanics and Geotechnical Engineering. 2009, pp. 5-9.

[12] P. Thurlow and R. K. Lipscombe. "The Use of Shape Accel Arrays (SAAs) for Measuring Retaining Wall Deflection". In: Proceedings of the 39th Annual Conference on Deep Foundations. 2014. 
